\title{
Análise funcional de indivíduos com lombalgia ocupacional
}

\author{
Analysis functional in individuals with backache occupational low back pain \\ Análisis funcional de individuos con lumbalgia ocupacional
}

Recebido: 19/10/2021 | Revisado: 27/10/2021 | Aceito: 01/11/2021 | Publicado: 02/11/2021

\author{
Kellen Cristiny Martins de Oliveira \\ ORCID: https://orcid.org/0000-0001-7272-7434 \\ Centro Universitário da Amazônia, Brasil \\ E-mail: kellenoliveira078@gmail.com \\ Ingrid Alynne Brenna Nathacha Da Silva Lemos \\ ORCID: https://orcid.org/0000-0001-6056-5845 \\ Centro Universitário da Amazônia, Brasil \\ E-mail: alynnelemos1@gmail.com.br \\ Wanderson Fernandes Silva \\ ORCID: https://orcid.org/0000-0001-9389-5795 \\ Centro Universitário da Amazônia, Brasil \\ E-mail: wandersonfsm@outlook.com
}

\begin{abstract}
Resumo
A lombalgia ocupacional é um problema que acomete trabalhadores que permanecem por longas horas em sedestação, afeta principalmente aqueles em idade produtiva. Objetivo: analisar a funcionalidade de indivíduos com lombalgia ocupacional. Metodologia: trata-se de um estudo de caso, descritivo e quantitativo, que foi realizado com 6 indivíduos de ambos os gêneros com idade entre 25 e 50 anos, foram utilizadas a escala visual análoga, questionário Nórdico de sintomas osteomusculares, questionário de Roland-Morris e avaliação fisioterapêutica. Resultados: a média de idade

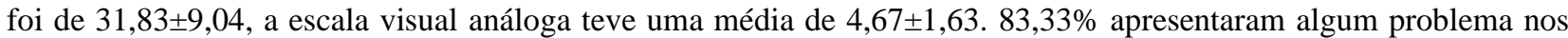
últimos 12 meses e 66,67\% manifestaram dor lombar nos últimos 7 dias, no questionário de Roland Morris obteve-se a pontuação de $2,33 \pm 2,42$, na análise de amplitude de movimento para flexão, foi possível observar a média de $86,67 \pm 9,83$, em relação a amplitude de extensão a média foi de $26,67 \pm 5,16$. Quando é analisado o grau de força

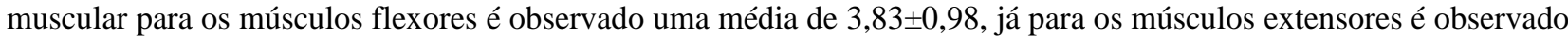

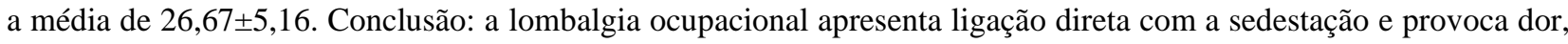
fraqueza muscular e encurtamento, alterando assim sua funcionalidade.
\end{abstract}

Palavras-chave: Lombalgia; Postura sentada; Doenças ocupacionais.

\begin{abstract}
Occupational low back pain is a problem that affects workers who remain seated for long hours, mainly affecting those of working age. Objective: to analyze the functionality of individuals with occupational low back pain. Methodology: this is a case study, descriptive and quantitative, which was carried out with 6 individuals of both genders, aged between 25 and 50 years, using the visual analogue scale, Nordic musculoskeletal symptoms questionnaire, Roland-Morris questionnaire and physical therapy assessment. Results: the mean age was 31.83 \pm 9.04 , the visual analogue scale had a mean of $4.67 \pm 1.63 .83 .33 \%$ had some problem in the last 12 months and $66.67 \%$ had low back pain in the last 7 days. The Roland Morris questionnaire obtained a score of $2.33 \pm 2.42$ in the range of motion analysis for flexion, it was possible to observe an average of $86.67 \pm 9.83$, in relation to the extension amplitude the average was $26.67 \pm 5.16$. When the degree of muscle strength for the flexor muscles is analyzed, an average of $3.83 \pm 0.98$ is observed, while the extensor muscles have an average of $26.67 \pm 5.16$. Conclusion: occupational low back pain is directly linked to sitting and causes pain, muscle weakness and shortening, thus altering its functionality.
\end{abstract}

Keywords: Low back pain; Sitting posture; Occupational diseases.

\section{Resumen}

La lumbalgia ocupacional es un problema que afecta a los trabajadores que permanecen sentados durante largas horas, afectando principalmente a los que están en edad de trabajar. Objetivo: analizar la funcionalidad de individuos con lumbalgia ocupacional. Metodología: se trata de un estudio de caso, descriptivo y cuantitativo, que se realizó con 6 individuos de ambos sexos, con edades comprendidas entre 25 y 50 años, utilizando la escala analógica visual, el cuestionario de síntomas musculoesqueléticos nórdicos, el cuestionario de Roland-Morris y la valoración de fisioterapia. Resultados: la edad media fue 31,83 \pm 9,04, la escala visual analógica tuvo una media de 4,67 $\pm 1,63$. El $83,33 \%$ tuvo algún problema en los últimos 12 meses y el 66,67\% tuvo lumbalgia en los últimos 7 días. El cuestionario de Roland Morris obtuvo una puntuación de 2,33 $\pm 2,42$ en el análisis de rango de movimiento para flexión, se pudo observar un promedio de $86,67 \pm 9,83$, en relación a la amplitud de extensión el promedio fue de 
$26,67 \pm 5,16$. Cuando se analiza el grado de fuerza muscular de los músculos flexores se observa un promedio de 3,83 $\pm 0,98$, mientras que para los músculos extensores se observa un promedio de $26,67 \pm 5,16$. Conclusión: la lumbalgia ocupacional está directamente relacionada con la posición sentada y provoca dolor, debilidad y acortamiento muscular, alterando así su funcionalidad.

Palabras clave: Lumbalgia; Postura sentada; Enfermedades profesionales.

\section{Introdução}

Com o advento da tecnologia o mundo mudou seu meio de trabalho e como consequência a utilização de computadores e outros aparelhos eletrônicos ficaram mais comuns, levando trabalhadores a passar mais tempo em sedestação, isso fez com que diversos países desenvolvidos e subdesenvolvidos passassem a ter gastos grandiosos com tratamentos musculoesqueléticos de colaboradores, em especial a lombalgia (Jung et al., 2021; Castillo et al., 2020).

Conforme Macedo (2011), a lombalgia é designada como uma dor que ocorre na coluna vertebral lombar que está presente entre as vértebras L1 e L5. A dor lombar apresenta um índice elevado, cerca de 70 a $80 \%$ da população em idade produtiva desenvolverá lombalgia em um determinado momento da vida. Ela pode ser classificada em início agudo com duração de quatro semanas, evoluir para subagudo com período de quatro a doze semanas e se tornar crônica com duração maior que três meses (Varrassi et al., 2021).

A posição sentada pode se tornar desconfortável se permanecida por longos períodos e ocasionar dores na coluna vertebral e em outras regiões do corpo, além de tensão e espasmos musculares (Allegri et al., 2016). De acordo com o Manual de aplicação da norma regulamentadora $\mathrm{N}^{\circ} 17$ de 2002, a posição de sedestação leva a complicações como o sedentarismo, gera compressão dos discos localizados entre as vértebras, compressão de ligamentos, tendões, bursas e aumento da cifose e lordose. Os estudos de Becker et al. (2018), mostraram que o hábito de permanecer longas horas em sedestação vai muito além de perturbações musculoesqueléticas como dores em região de lombar e membros inferiores, ela acomete também os níveis de aquisição de conhecimento.

É comum que com o passar do tempo em posição sentada o indivíduo altere a posição para uma postura mais ereta diminuindo a lordose, como se estivesse deitado ou uma postura mais curvada onde ocorre o aumento da cifose torácica. A posição na qual o indivíduo fica mais deitado causa fadiga da musculatura especialmente do músculo oblíquo interno e transverso do abdômen. Esses músculos possuem a função de gerar estabilidade da coluna o que equilibra as forças compressivas, quando o indivíduo fica em uma posição mais curvada esses mesmos músculos sofrem uma diminuição da atividade (Jung et al., 2021; Waongenngarm et al., 2016).

As dores lombares não são causadas apenas por posturas inadequadas ou por tempos prolongados em sedestação, o ambiente em que o colaborador está inserido influencia, de forma direta, a exemplos os assentos. $\mathrm{O}$ assento salli mantém o ângulo ideal da lordose, porém, o assento com suporte isquiático provoca menos algias, mas vale ressaltar que ambos geram incômodos após longas horas de trabalho sentado (Santos \& Araújo, 2017).

As normas regulamentadoras do trabalho NR-17, asseguram que o ambiente de trabalho seja adaptado conforme a biomecânica do colaborador, na norma 17.3.3 é necessário o ajuste do local conforme a estatura do trabalhador, os assentos precisam possuir uma forma convexa e as costas precisam manter um ângulo ideal para a lombar.

Além da adaptação do ambiente de trabalho, a ginástica laboral realizada por meio de alongamentos durante os intervalos no trabalho gera inúmeros benefícios para os colaboradores como a diminuição das dores e correção do hábito postural (Candotti et al., 2011). A medida que a ginástica laboral não apresenta mais efeitos significativos sobre as algias da coluna vertebral, a Fisioterapia entra com um papel de suma importância, com técnicas de reabilitação para a diminuição das dores, aumento da amplitude de movimento articular, alongamento dos tecidos que apresentam encurtamento e melhora também o condicionamento físico (Natuor, 2004). 
Segundo o IBGE, em 2019, 34,3 milhões de brasileiros apresentaram dor na coluna vertebral, destes, 2,5\% foram diagnosticados com distúrbio osteomuscular relacionados ao trabalho (DORT). Em 2016 o Brasil retirou dos cofres públicos $\mathrm{R} \$ 298$ milhões para diagnosticar e tratar doenças relacionadas à coluna vertebral (Alessandro, 2019). Com isso, o presente estudo tem como objetivo analisar a funcionalidade de indivíduos com lombalgia ocupacional que trabalham por longas horas em sedestação.

\section{Metodologia}

Este estudo foi aprovado pelo Comitê de ética em pesquisa, Instituto Campinense de Ensino Superior - ICESUNAMA (060316/2021) e respeita os princípios e diretrizes da resolução ${ }^{\circ} 466$ de 12 de dezembro de 2012 do Conselho Nacional de Saúde que envolve pesquisa com seres humanos.

O presente estudo pode ser classificado em: estudo de caso, descritivo e quantitativo (Fontelles et al., 2009). Realizado com 6 participantes, com idade entre 25 e 50 anos, de ambos os gêneros. Para análise de dados, recorreu-se ao software BioEstat 5.0. A pesquisa foi realizada em uma empresa privada do Município de Santarém-PA, em sala apropriada e reservada, nela continham 1 maca, 1 goniômetro, 1 medidor de pressão arterial digital e um aparelho de eletroestimulação. Os indivíduos, incluídos no grupo, deveriam apresentar idade entre 25 e 50 anos, trabalhar em sedestação no mínimo 5 dias por semana com carga horária mínima de 6 horas diárias de trabalho, bem como ter assinado o Termo de Consentimento Livre e Esclarecido (TCLE), foram excluídos grávidas ou indivíduos com Covid-19.

A pesquisa foi dividida em duas fases, na primeira foi realizada a apresentação do Termo de Consentimento Livre e esclarecido (TCLE) para que os participantes tirassem suas dúvidas acerca do projeto e estivessem cientes que teriam suas informações e imagens preservadas, em seguida, foi aplicada a Escala Visual Análoga (EVA), questionário Nórdico de sintomas osteomusculares e, por fim, o questionário de Roland-Morris. A segunda fase foi composta por uma avaliação fisioterapêutica que visava a análise da amplitude de movimento e grau de força muscular dos músculos flexores e extensores.

A escala visual análoga (EVA) é amplamente utilizada pelos profissionais da área da saúde para mensurar o nível de dor, ela varia em uma escala de 0 a 10, sendo de 0 a 2 ausência de dor, 3 a 7 dor moderada e 8 a 10 dor muito intensa (Heinen et al., 2016).

O questionário Nórdico de sintomas osteomusculares serve para analisar os sintomas dos participantes da pesquisa, ele é definido por um desenho do corpo humano com setas que sinalizam cada região, a amostra marcou sim ou não para os sintomas relacionados a coluna vertebral lombar nos últimos 12 meses e 7 dias (Santos et al., 2015).

O questionário de Roland-Morris é utilizado para avaliar a limitação diária de indivíduos que apresentam lombalgia, é composto por 24 frases que retratam limitações na vida diária seja para dormir se vestir ou trabalhar, o resultado será a soma dos itens marcados com X, quanto mais frases marcadas maiores são as dificuldades (Sardá et al., 2010).

A avaliação da amplitude de movimento foi feita utilizando um goniômetro que serve para determinar o grau de amplitude de movimento de uma determinada articulação, para que fosse mensurado o grau de flexão o indivíduo ficou em posição ortostática, o eixo do goniômetro foi localizado na espinha ilíaca antero-superior (EIAS), enquanto que o braço fixo em direção ao solo e o móvel em direção a linha axilar. Para a verificação do grau de extensão o indivíduo voltou à posição inicial, o eixo do goniômetro ficou na mesma posição, citado anteriormente e o braço fixo recolocado em direção ao côndilo lateral do fêmur e o móvel em direção a linha axilar do tronco (Palmer \& Epler, 2013; Marques, 2003).

Para determinar o grau de força muscular dos flexores e extensores da coluna vertebral lombar, foi utilizado uma escala que vai de 0 a 5 onde zero significa sem de contração muscular e 5 contração muscular com resistência máxima, para flexão os indivíduos ficaram em decúbito dorsal enquanto que para extensão em decúbito ventral (Palmer \& Epler, 2013; Conceição, 2016). 
Os dados foram coletados e tabulados na planilha do software Excel (Microsoft ${ }^{\circledR}$ - EUA) de forma codificada entre os participantes, foram tratados de maneira descritiva utilizando valores, números em média e desvio padrão.

\section{Resultados}

O presente estudo teve como objetivo primário analisar a funcionalidade de indivíduos com lombalgia ocupacional, para tanto, 6 sujeitos participaram do estudo, 4 do sexo masculino e 2 do sexo feminino, com idade média de 31,83 $\pm 9,04$, variando de 23 a 43 anos. Na análise de dor realizada por meio da escala visual analogia, foi possível observar menor

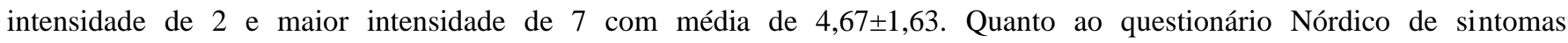
osteomusculares para dor lombar, verificou-se que nos últimos 12 meses, 83,33\% dos participantes apresentaram algum problema como dor, formigamento ou dormência lombar, quanto aos impedimentos de realizar atividades normais durante os últimos 12 meses, nenhum participante relatou apresentar intercorrência. Já nos últimos 12 meses, 16,66\% dos participantes buscaram um profissional da saúde e nos últimos 7 dias 66,67\% manifestaram dor lombar.

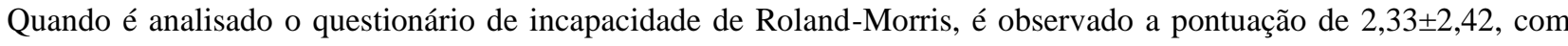
menor média de 0 e maior média de 7. No item 2 (Eu mudo de posição frequentemente para tentar aliviar minha coluna) 4 participantes se enquadram, no item 3 (Eu ando mais lentamente do que o meu normal por causa de minha coluna) somente 1 participante se identificou, já no item 6 (Por causa de minhas costas, eu deito para descansar mais frequentemente) apenas 2 participantes marcaram, no item 10 (Eu fico de pé por períodos curtos, por causa de minhas costas) somente 1 participante se enquadrou, já no item 11(Por causa de minhas costas, eu procuro não me curvar ou agachar) só 2 pacientes se encaixaram, quanto ao item 13 (Minhas costas doem a maior parte do tempo) teve a pontuação de 1 participante, assim como no item 21 (Eu evito trabalhos pesados em casa por causa de minhas costas) permaneceu 1 participante, no item 22 (Devido a minha dor nas costas fico mais irritado e de mau humor com as pessoas) manteve-se com 1 participante e no item 24 (Fico na cama a maior parte do tempo por causa de minhas costas) somente 1 participante se identificou, os demais itens não tiveram a presença de participantes.

$\mathrm{Na}$ análise de amplitude de movimento, foi possível observar menor grau de amplitude de flexão de $70^{\circ}$ e maior de $90^{\circ}$ com média de $86,67 \pm 9,83$, em relação à amplitude de extensão o menor grau observado foi de $20^{\circ}$ e maior de $30^{\circ}$, com

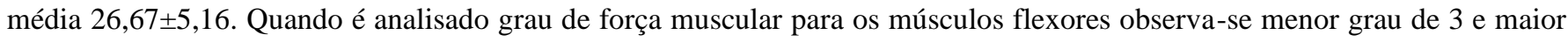

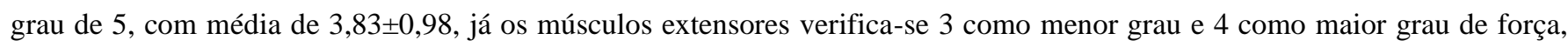
com média de $26,67 \pm 5,16$. Os dados referentes a dor, amplitude de movimento e força de músculos de coluna vertebral podem ser observados na Tabela 1.

Tabela 1. Média aritmética referente ao grau de força muscular e ADM* para os músculos flexores e extensores de coluna vertebral lombar e grau de dor referida.

\begin{tabular}{ccccc}
\hline & Força muscular & ADM* & Dor (EVA*) & Roland-Morris \\
Flexão & $3,83 \pm 0,98$ & $86,67 \pm 9,83$ & $4,67 \pm 1,63$ & $2,33 \pm 2,42$ \\
Extensão & $3,83 \pm 0,41$ & $26,67 \pm 5,16$ & & \\
& & & & \\
\hline
\end{tabular}

*ADM: amplitude de movimento; EVA: escala visual analógica. Fonte: Autores. 


\section{Discussão}

O presente estudo teve como objetivo analisar a funcionalidade de indivíduos com lombalgia ocupacional, para tanto foi composto por 6 participantes de ambos os sexos com idade entre 23 a 43 anos, dados que se encaixam nos estudos de Lopes

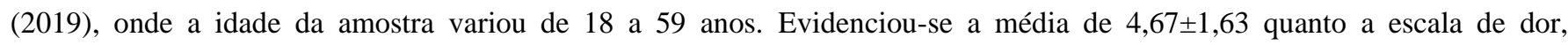
caracterizando-se como dor moderada. Em um estudo realizado na cidade de Santa Catarina com 8 funcionários, teve a média de $5 \pm 2$ o que corrobora com o presente estudo (Pivetta \& Vey, 2014).

No questionário Nórdico de Sintomas Osteomusculares observa-se que, nos últimos 12 meses, 83,33\% dos participantes apresentaram alguma dor, sendo que 16,66\% procuraram um profissional da área da saúde, assim como 66,67\% manifestaram dor lombar nos últimos 7 dias anteriores à pesquisa, entretanto, não houve afastamento do trabalho nos últimos 12 meses. Tal dado encontra-se de acordo com o estudo de Figueira (2018), que de 53 trabalhadores do setor de administração $51 \%$ apresentaram dor nos últimos 12 meses, 25,92\% procuraram auxílio médico e somente $2 \%$ foram afastados, consequências essas da posição sentada, que permanecida por longas horas gera sobrecarga da coluna vertebral lombar além de alteração musculoesquelética tendo em vista que não ocorre dissipação do peso, sendo ele sustentado somente pela tuberosidade isquiática (Silva \& Silva 2021).

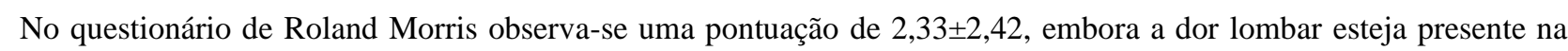
amostra estudada ela não acarreta limitações severas, assim como em um estudo realizado com 22 pessoas, onde o comprometimento variou entre 18,8\% a 6,3\% (Machado, 2021). Deve-se, a idade da amostra ser mais jovem e possuir uma vida mais ativa, apesar da lombalgia restringir de forma moderada ela esporadicamente causa impedimento total para a realização de trabalhos, afazeres domésticos ou de lazer (Barros et al., 2020; Zanatelli et al., 2021).

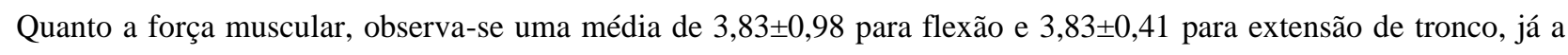
amplitude de movimento mostrou-se a média de $86,67 \pm 9,83$ para flexão e $26,67 \pm 5,16$ para extensão, resultados esses que se assemelham com os estudos de Santos, et al. (2015), que de 149 prontuários avaliados, a média apresentou amplitude de movimento e força muscular diminuída, uma vez que a posição sentada diminui a atuação dos grupos musculares e consequentemente leva a uma fraqueza da musculatura e um encurtamento gradual do tronco alterando assim sua função (Fujitani et al., 2021; Soares et al., 2013; Silva \& Dande, 2017).

\section{Conclusão}

Com base nos resultados encontrados e na literatura vigente, evidencia-se que a lombalgia ocupacional apresenta ligação direta com a sedestação e provoca dor, fraqueza muscular e encurtamento, alterando assim sua funcionalidade. No entanto, por tratar-se de um estudo de caso os resultados apresentam certa limitação, sugere-se a elaboração de estudos com amostras maiores e metodologia mais elaborada com enfoco em saios clínicos controlados randomizados a fim de contribuir, ainda mais, para a elucidação da relação entre a lombalgia ocupacional e o trabalho em sedestação.

\section{Referências}

Alessandro, M. D. (2019). SUS faz uso excessivo de exames de imagem no diagnóstico de problemas na coluna, revela artigo. https://www.unbciencia.unb.br/biologicas/63-fisioterapia/622-sus-faz-uso-excessivo-de exames-de-imagem-no-diagnostico-de-problemas-na-coluna-revelaartigo

Allegri, M., Montella, S., Salici, F., Valente, A., Marchesini, M., Compagnone, C., Baciarello, M., Manferdini, M. E., \& Fanelli, G. (2016). Mechanisms of low back pain: a guide for diagnosis and therapy [version 2; peer review: 3 approved]. Journal F1000Research. https://doi.org/10.12688/f1000research.8105.2

Baker, R., Coenen, P., Howie, E., Williamson, A., \& Straker, L. (2018). The Short Term Musculoskeletal and Cognitive Effects of Prolonged Sitting During Office Computer Work. International Journal of Environmental Research and Public Health. $10.3390 /$ ijerph15081678

Barros, F. P., Bastos, R. F. N., \& Lopes, R. L. (2020). Prevalência de lombalgia em motoristas de caminhão da rede privada. Revista das Ciências da Saúde e Ciências aplicadas do Oeste Baiano-Higia, 5 (1), 20-35. 
Candotti, C. T., Stroschein, R., \& Noll, M. (2011). Efeitos da ginástica laboral na dor nas costas e nos hábitos posturais adotados no ambiente de trabalho. Revista Brasileira de Ciências do Esporte, 33 (3), 699-714.

Castillo-ante, L., Ordoñez-Hernández, C., \& Calvo-Soto, A. P. (2020). Carga física, estrés y morbilidad sentida osteomuscular en trabajadores administrativos del sector público. Universidad y Salud, 22 (1), 17-23. https://doi.org/10.22267/rus.202201.170

Conceição, F. F. (2016). Avaliação cinético-funcional. SESES.

Figueira, C. F. (2018). Prevalência de dor lombar em profissionais de setor administrativo que atuam na postura sentada (Trabalho de Conclusão de Curso). Faculdade União das Américas - UNIAMÉRICA, Foz do Iguaçu, Brasil.

Fontelles, M. J., Simões, M. G., Farias, S. H., \& Fontelles, R. G. S. (2009). Metodologia da pesquisa científica: diretrizes para a elaboração de um Protocolo de pesquisa. Revista paraense de medicina, 23 (3), 1-8.

Fujitani. R., Jiroumaru, T., Noguchi, S., Wachi, M., Ohnishi, H., Suzuki, M., \& Ozawa, T. (2021). Effect of low back pain on the muscles controlling the sitting posture. J. Phys. Ther. Sci, 33, 295-298.

Heinen, A. C., Goulart, C. L., Soudbrack, A. C., Fleig, T. C. M., \& Silva, A. L. G. (2016). Avaliação da dor como sinal vital: uma escolha profissional de intervenção fisioterapêutica. Revista Pesquisa em Fisioterapia, 6 (4). 10.17267/2238-2704rpf.v6i4.935

IBGE- Instituto Brasileiro De Geografia E Estatística. Censos 2019. Pesquisa Nacional de Saúde. Percepção do estado de saúde, estilos de vida, doenças crônicas e saúde bucal. Rio de Janeiro: IBGE 2020

Jung, K. Sim., Jung, J. H., Sung, T., \& Cho, H. Y. (2021). Effects of Prolonged Sitting with Slumped Posture on Trunk Muscular Fatigue in Adolescents with and without Chronic Lower Back Pain. Medicina, 57 (3). doi: https://doi.org/. https://dx.doi.org/ 3390/medicina 57010003

Lopes, A. R. (2019). Prevalência e fatores associados a sintomas osteomusculares em profissionais que trabalham predominantemente na postura sentada (Tese de doutorado). Universidade de São Paulo, Ribeirão Preto, Brasil.

Macedo, D. D. P. (2011). Lombalgias. Revista Ciência e Cultura, 63 (2), 42-44. http://dx.doi.org/10.21800/S0009-67252011000200013

Machado, G. Z. (2021). Efeitos do treinamento de força em sujeitos com lombalgia (Trabalho de Conclusão de Curso). Universidade Regional do Noroeste do Estado do Rio Grande do Sul, Santa Rosa, Brasil.

Manual de aplicação da norma regulamentadora $\mathrm{N}^{\circ}$ 17. (2a ed.).

Marques, A. P. (2003). Manual de goniometria (2a ed.). Manole.

Natuor, J. (2004). Coluna vertebral: conhecimentos básicos (2a ed.). Etcetera.

Palmer, M. L., \& Epler, M. E. (2013). Fundamentos e técnicas de avaliação musculoesquelética (2a ed.). Guanabara Koogan.

Pivetta, H. M. F., \& Vey, A. P. Z. (2014). Frequência de dor lombar em operadores de telemarketing em Santa Maria. Revista Saúde, 40 (2), 141-146.

Santos, C. M. E., \& Araújo, R. C. (2017). Assessment of changes in spine curvatures and the sensations caused in three different types of working seats. Revista educação física, 23(3). https://doi.org/http://dx.doi.org/10.1590/S1980-6574201700030002

Santos, I. M., Santos, J. W., Alsina, O. L. S., \& Monteiro, L. F. (2015). Aplicação do questionário Nórdico musculoesquelético para estimar a prevalência de distúrbios osteomusculares relacionados ao trabalho em operárias sob pressão temporal. In: Encontro Nacional de Engenharia de Produção - Enegep Fortaleza, CE, Brasil. https://ri.ufs.br/bitstream/riufs/8599/2/AplicacaoQuestionarioNordicoMuscoesqueletico.pdf

Santos, J. K. V., Junior Gomes, V. F. F., Souza, A. S., Farias, N. S., Marques, S. S., \& Costa, J. M. (2015). Perfil sócio-demográfico e físico-funcional de pacientes com lombalgia atendidos em Manaus/AM. Revista Dor, 16 (4), 272-5.

Sardá Júnior, J. J., Nicholas, M. K., Pimenta, C. A. M., Asghari, A., \& Thieme, A. L. (2010). Validação do Questionário de Incapacidade Roland Morris para dor em geral, Revista Dor, 11 (1), 28-36.

Silva, J. C. A., \& Dande, J. V. D. (2017). Prevalência de dores osteomusculares em colaboradores de escritórios de contabilidade (Trabalho de conclusão de curso). Centro Universitário do Sul de Minas - UNIS-MG, Minas Gerais, Brasil.

Silva, T. A. M., \& Silva, R. J. R. (2021). Análise dos riscos ergonômicos e posturais dos funcionários da secretaria acadêmica de uma faculdade privada de saúde do Recife (Trabalho de Conclusão de Curso). Faculdade Pernambucana de Saúde, Recife, Brasil.

Soares, R. S., Silva, J. A. M. G., Silva, M. G. M. G., \& Navega. M. T. (2013). Relação entre incapacidade funcional, amplitude de movimento e dor em indivíduos com e sem lombalgia, 11 (51), 43-47.

Varrassi, G., Moretti, B., Pace, M. C., Paolo, E., \& Iolascon, G. (2021). Common Clinical Practice for Low Back Pain Treatment: A Modified Delphi Study. Pain and Therapy. https://doi.org/10.1007/s40122-021-00249-w

Waongenngarm, P., Rajaratnam, B., \& Janwantanakul, P. (2016). Internal Oblique and Transversus Abdominis Muscle Fatigue Induced by Slumped Sitting Posture after 1 Hour of Sitting in Office Workers. Safety and Health at Work, 7 (1), 49-54. http://dx.doi.org/10.1016/j.shaw.2015.08.001

Zanatelli, M. M., Guimarães, A. V., Storte, G. R., Velloso, N., Emidio, M. V., Peruzzetto, M. C., \& Bastos, P. A. S. (2021). Prevalência de dor lombar no Porto de trabalhadores Santos. Revista Brasileira Medicina do Trabalho, 19 (2), 173-180. 